\title{
Latency, stochastic dominance, and laterality effects ${ }^{1,2,3}$
}

WILLIAM M. PETRUSIC ${ }^{4}$

UNIVERSITY OF MICHIGAN

Definitions of transitivity of choice are provided for latency of choice, and it is shown that in both preferential and judgmental choice behavior this definition accurately reflects probabilistic transitivity of choice. It is shown, moreover, that both temporal and probabilistic transitivity are subject to laterality effects as predicted by Coombs' unfolding theory.

This report deals with transitivity of choice in both preferential and judgmental choice behavior. It also examines the effects of laterality (see Coombs, 1958, 1964 for complete discussion of laterality) on strong stochastic transitivity. Finally, conditions of transitivity are defined on latency of choice and the effects of laterality on this condition of transitivity are also investigated.

Prior to indicating the constraints imposed on choice behavior by the various degrees of transitivity we first define:

(1) Stochastic Dominance: $S_{i}(S D) S_{j} \equiv \operatorname{Pr}\left[S_{j}>S_{j}>P \operatorname{Pr}\left[S_{j}>S_{j}\right]\right.$ (where $S_{j}>S_{j}$ denotes that stimulus $S_{i}$ is ordered above stimulus $S_{j}$ ). $S_{j}$ is called stochastically dominant (SD), and $S_{j}$ is called stochastically non-dominant. Whenever, $\operatorname{Pr}\left[S_{j}>S_{j}\right]=$ $\operatorname{Pr}\left[S_{j}>S_{i}\right]$ then $S_{i}$ and $S_{j}$ are called stochastically equivalent (SE).

The various conditions of transitivity can now be stated as follows:

(2) Strong-Stochastic Transitivity (SST) Given $S_{j}(S D) S_{j}$ and $S_{j}(S D) S_{k}$ then $\operatorname{Pr}\left[S_{j}>S_{k}\right]>\max .\left\{\operatorname{Pr}\left[S_{j}>>S_{j}\right], \operatorname{Pr}\left[S_{j}>S_{k}\right]\right\}$ : (3) Moderate-Stochastic Transitivity (MST) Given $S_{j}(S D) S_{\text {j }}$ and $S_{j}(S D) S_{k}$ then $\operatorname{Pr}\left[S_{j} \cdot>S_{k}\right]>\min .\left\{\operatorname{Pr}\left[S_{i}>>S_{j}\right], \operatorname{Pr}\left[S_{j}>S_{k}\right]\right\}$. (4) Weak-Stochastic Transitivity (WST) Given $S_{j}(S D) S_{j}$ and $S_{j}(S D) S_{k}$ then $S_{j}(S D) S_{k}$.

Corresponding to (2) and (3), respectively, transitivity conditions on latency of choice can be defined as follows: (5) Strong-Temporal Transitivity (STT) Given $S_{j}(S D)$ and $S_{i}(S D) S_{k}$ then $\left[\left(S_{j}>S_{k}\right)<\min .\left\{L\left(S_{j}>S_{j}\right), L\left(S_{j}>S_{k}\right)\right\}\right.$ (where $\left[\left(S_{j}>S_{k}\right)\right.$ denotes the mean latency of the set of choices when $S_{j}$ is ordered above $S_{j}$ ).

(6) Moderate-Temporal Transitivity (MTT) Given $S_{j}(S D) S_{j}$ and $S_{j}(S D) S_{k}$ then $\left[\left(S_{j}>S_{k} \bar{L}<\max .\left\{\left[\left(S_{i}>S_{j}\right), \bar{L}\left(S_{j}>S_{k}\right)\right\}\right.\right.\right.$

The following possible relationships will be investigated in this paper:

(R1): SST $\longrightarrow$ STT

(R2): MST $<\longrightarrow$ MTT

A preliminary analysis of data obtained from an experiment conducted by the author suggested that the above relationships held strongly and that SST and STT were subject to laterality effects as predicted. In this experiment the stimuli were two outcome gambles (for a description of this experiment see Petrusic, 1966), and the Ss were required to indicate their preferences and to judge which gamble was riskier in each pair by the method of paired comparisons. It turned out that each S's preference ordering would be generated by folding his risk scale and, thus, it was possible to determine the effects of laterality on SST and STT. Since these results are based on only two Ss, and since the determination of laterality depending on a $S$ folding his own risk scale at the preferred amount of perceived risk, the results may have been somewhat tenuous. Thus, a second set of data, taken from Greenberg (1961) was utilized for testing (R1) and (R2) and for determining whether SST and STT are subject to laterality effects. These data were selected because a clearly defined $\mathrm{J}$-scale existed from which preference orderings could be generated. Consequently, the effects of laterality could be established with greater confidence than in the author's experiment.

Method

This experiment is described in its entirety in Greenberg (1961). Four male undergraduate students at the University of Michigan served as Ss in this experiment.

Six shades of gray were used as stimuli and were constructed from neutral density filters. Stimuli were presented in pairs under the following four sets of instructions.

B1. "Choose that patch which appears to be the darker of the two";

A2. "... choose that patch which you prefer as the better representative of a good medium gray";

A3. "...choose that patch which you prefer as the better representative of a good light gray";

A4. "... choose that patch which you prefer as the better representative of a good dark gray."

Stimulus pairs were projected on a screen and Ss made their choices by pressing one of two keys. The S's response and its latency were automatically recorded on an IBM card.

A total of fifty replications on each stimulus pair was obtained under each of the four sets of instructions.

\section{Results}

Stochastic and Temporal Transitivity: (R1) and (R2)

The data for all four Ss and all three Task A tasks have been combined since there were no differences between Ss with respect to the degrees to which S'S and STT held, and since too few triples for each laterality class were obtained from each S. SST was determined from the summary over all replications, and STT was determined from the mean dominant 
latencies over all replications.

The hypothesis of independence between conditions (2) and (5) is rejected $\left(X^{2}=58.35 ; d f=1 ; p<0.001\right)$ for the Task A data. For 199 of the 240 triples, or in 82.9 percent of the cases, SST and STT simultaneously hold or fail to hold for the Task A data. MST and MTT simultaneously hold in almost every case. For the Task B data 76 of the 80 , or 95.9 percent of the triples, simultaneously satisfy both SST and STT. All triples satisfy MST and MTT.

Laterality Effects

Table 1 presents the frequency of cases in which SST and STT simultaneously hold or fail to hold according to type of triple. No bilateral split triples violate SST and in one case of 9 STT is violated. For unilateral triples SST is satisfied in 57 of 62 cases and in 59 cases STT is satisfied. Thus a total of 66 of 71 triples, which are bounded by a unilateral pair of stimuli, satisfy SST and 67 satisfy STT. However, for the triples where an embedded pair of stimuli is bounded by a bilateral pair of stimuli (bilateral adjacent triples) SST holds in 21 of 45 cases and STT in 16 cases. This difference between type of bounding pair is statistically significant $\left(X^{2}=29.06 ; d f=1 ; p<0.001\right)$ for both SST and STT.

MST is of course not differentially satisfied by the various triples.

\section{Discussion}

The results strongly suggest that response latency is an extremely sensitive alternative response measure in that it reflects relatively accurately stochastic transitivity of choice and the effects of laterality on SST. One important implication of (R1) and (R2) is that response latency from a single replication may possibly

Table 1. Effects of laterality on SST and STT.

Summary over all replications, tasks and subjects.

Original data taken from Greenberg, M. (1961)

\begin{tabular}{|c|c|c|c|c|c|c|}
\hline \multirow[b]{2}{*}{$\begin{array}{l}\text { Type of } \\
\text { Bounding Stimulus } \\
\text { Pair }\end{array}$} & \multirow[b]{2}{*}{$\begin{array}{l}\text { Type of } \\
\text { Triple }\end{array}$} & \multirow[b]{2}{*}{$\mathrm{s}$} & \multicolumn{4}{|c|}{ SST } \\
\hline & & & Sat. & $\begin{array}{l}\text { Sat. } \\
14\end{array}$ & $\begin{array}{c}\text { Not. Sat. } \\
2\end{array}$ & $\begin{array}{c}\text { Tota } \\
16\end{array}$ \\
\hline \multirow[t]{2}{*}{ Bilateral } & Bilateral & $\begin{array}{l}T \\
T\end{array}$ & Not.Sat. & 7 & 22 & 29 \\
\hline & Adjacent & & Total & 21 & 24 & 45 \\
\hline & & & \multicolumn{4}{|c|}{ SST } \\
\hline & & S & & $\begin{array}{c}\text { Sat. } \\
56\end{array}$ & $\begin{array}{c}\text { Not. Sat. } \\
3\end{array}$ & $\begin{array}{c}\text { Tota } \\
59\end{array}$ \\
\hline & Unilateral & $\begin{array}{l}T \\
T\end{array}$ & Not. Sat. & .1 & 2 & 3 \\
\hline \multirow[t]{5}{*}{ Unilateral } & & & Total & 57 & 5 & 62 \\
\hline & & & \multicolumn{4}{|c|}{ SST } \\
\hline & & $\mathrm{S}$ & Sat. & 8 & 0 & 8 \\
\hline & $\begin{array}{l}\text { Bilateral } \\
\text { Split }\end{array}$ & $\begin{array}{l}T \\
T\end{array}$ & Not.Sat. & . 1 & 0 & 1 \\
\hline & & & Total & 9 & 0 & 9 \\
\hline
\end{tabular}

be used as a technique for assessing SST and MST where it is not possible to obtain several independent replications of paired comparison choices.

Luce's (1959) criticism that in the Coomb's (1958) study SST was not determined from paired-comparison choices but from the decomposed rankings of Ss of four stimuli (quads) is not applicable in this study. Nevertheless strong transitivity is violated as predicted by unfolding theory on the basis of both inconsistency of choice and latency of choice.

It should be noted that the transitivity conditions as defined in this paper are not independent of SD. In this study the latency data were primarily viewed as an alternative response measure, which could be utilized to confirm or disconfirm the results on SST and laterality effects. If transitivity of latency is to be defined independently of SD then such definitions would take the following form, after first defining temporal dominance:

(7) Temporal Dominance (TD) $S_{j}(T D) S_{j}=\bar{L}\left(S_{j}>S_{j}\right)<\bar{L}\left(S_{j}>S_{j}\right)$

(8) Weak Temporal Transitivity (WTT): Given $S_{i}(T D) S_{j}$, and $S_{j}(T D) S_{k}$ then $S_{i}(T D) S_{k}$.

(9) Moderate Temporal Transitivity (MTT): Given $S_{i}($ TD)S and $S_{j}(T D) S_{k}$ then $\left[\left(S_{j}>S_{k}\right)<\max .\left\{L\left(S_{j}>S_{j}\right), \bar{L}\left(S_{j} \cdot>S_{k}\right)\right\}\right.$

(10) Strong Temporal Transitivity (STT): Given $S_{j}(T D) S_{j}$ and $S_{j}(T D) S_{k}$ then $\mathrm{L}\left(S_{i}>S_{k}\right)<\min \left\{L\left(S_{i}>S_{j}\right), L\left(S_{j}>S_{k}\right)\right\}$

Now, one can inquire whether the following interrelations hold:

(R3) WST $<\longrightarrow$ WTT

(R4) $\mathrm{MST}<\longrightarrow \mathrm{MTT}$

(R5) $\mathrm{SST}<\longrightarrow \mathrm{STT}$

Whether, these interrelations hold, however, now depends on whether SD $\longrightarrow$ TD holds, for if not, the orderings on a triple $\left(S_{i}, S_{j}, S_{k}\right)$ may be completely reversed. Moreover, the results of Petrusic (1966) suggest that whether these relations will hold may depend on the particular response set that is operating.

\section{References}

Coombs, C. H. On the use of inconsistency of preferences in psychological measurement. $J$. exp. Psychol., 1958, 55, 1-7.

Coombs, C. H. A theory of data. New York: Wiley, 1964.

Greenberg, M. Response latency as a test of mathematical modes for preference behavior. Unpublished Ph.D. dissertation. University of Michigan, 1961.

Luce, D. Individual choice behavior. New York: Wiley, 1959. Petrusic, W. M. The relationship between stochastic and temporal dominance under an accuracy set. Psychon. Sci., 1966, 5, 373-374.

\section{Notes}

1. This work was supported in part by U.S.P.H.S. Grants M.H. 04236(C1), and NIHTIGM 1231-01.

2. The author wishes to thank Dr. C. H. Coombs and Dr. Robyn M. Dawes for the encouragement and helpful criticisms.

3 . At the time of preparation of this report the author discovered that Gordon Bechtel at the Oregon Research Institute had independently arrived at similar definitions of transitivity of response latency.

4. Now at the Department of Psychology, University of British Columbia. 